\title{
On the Diameter of Plane Curves
}

\author{
SHIH-TSAI FENG ${ }^{1}$ and GEORG SCHUMACHER ${ }^{2}$ \\ ${ }^{1}$ Max-Planck-Institute for Mathematics in the Sciences (MIS), Inselstr. 22-26, \\ D-04103 Leipzig, Germany \\ ${ }^{2}$ Fachbereich Mathematik Und Informatik, Philipps-Universität, D-35032 Marburg, Germany \\ e-mail:schumac@mathematik.uni-marburg.de
}

(Received: 11 November 1997; accepted in final form: 1 September 1998)

\begin{abstract}
Recently, differential geometric properties of embedded projective varieties have gained increasing interest. In this note, we consider plane algebraic curves equipped with the Fubini-Study metric from $\mathbb{P}_{2}(\mathbb{C})$ and give an estimate for the diameter in terms of the degree, initiated in a paper by F. A. Bogomolov.
\end{abstract}

Mathematics Subject Classifiation (1991): 53A20.

Key words: diameter, plane curve, Fubini-study metric.

Recently, differential geometric properties of embedded projective varieties have gained increasing interest. In this note, we consider plane algebraic curves equipped with the Fubini-Study metric from $\mathbb{P}_{2}(\mathbb{C})$ and give an estimate for the diameter in terms of the degree, initiated in a paper by F. A. Bogomolov [2]. In particular, this paper implied that contrary to a general belief the diameter is not bounded from above. The result was extended by N. A'Campo [1]. The curvature had been explicitly computed by L. Ness [5]. Her results show the existence of areas of negative curvature and that the curvature is not bounded from below in the family of all embedded algebraic curves of a fixed degree. Using curvature when proving an estimate for the diameter requires a careful consideration of these areas. Bogomolov pointed out that the best estimate to expect is logarithmic, since Gromov's Betti number theorem implies a lower estimate for the diameter in the following sense: Under the restriction to curves, whose curvature is bounded from below by a number $-\kappa^{2}$, the diameter is bounded from below by $C \log (d) / \kappa$, where $C$ denotes a posivite constant. We use rather explicit methods to show the following result

THEOREM 1. The diameter of a plane algebraic curve $C \subset \mathbb{P}_{2}(\mathbb{C})$ of degree $d$, equipped with the Fubini-Study metric is bounded by $\left(2 d^{2}-2 d+1\right)\left(4 d^{2}+1\right) \cdot \pi$.

The theorem has immediate consequences for the diameter of complete intersections in $\mathbb{P}_{n}$. Our estimate seems to be also of interest in connection with results of Y. Yomdin [6] and M. Briskin-Y. Yomdin [3] in the area of polynomial control problems. 


\section{Preparations}

Our estimates will be based upon projections onto projective lines. Let $\left(x_{0}: x_{1}: x_{2}\right)$ denote homogeneous coordinates on $\mathbb{P}_{2}=\mathbb{P}_{2}(\mathbb{C})$. For $j=0,1,2$ and $\{j, k, \ell\}=\{0,1,2\}$ we consider the lines $L_{j}$ defined as zero sets $V\left(x_{j}\right)$ and the points $P_{j}$ defined as zero sets $V\left(x_{k}, x_{\ell}\right)$. Furthermore, we have the canonical projections $\pi_{j}: \mathbb{P}_{2} \backslash P_{j} \rightarrow L_{j}$, defined by omitting the $j$ th coordinate. The projective plane $\mathbb{P}_{2}$ and the lines $L_{j}$ resp. are equipped with the Fubini-Study forms $\omega_{\mathbb{P}_{2}}$ and $\omega_{\mathbb{P}_{1}}$ resp.

LEMMA 1. Let $\gamma:[0,1] \rightarrow \mathbb{P}_{2} \backslash\left\{P_{0}, P_{1}, P_{2}\right\}$ be a curve of class $C^{\infty}$. Then the length is estimated by $L(\gamma) \leqslant \sum_{j=0}^{2} L\left(\pi_{j} \circ \gamma\right)$, or equivalently $\omega_{\mathbb{P}_{2}} \leqslant$ $\sum_{j=0}^{2} \pi_{j}^{*}\left(\omega_{\mathbb{P}_{1}}\right)$. on $\mathbb{P}_{2} \backslash \bigcup L_{j}$.

Proof. With respect to inhomogenous coordinates $\left(1: x_{1}: x_{2}\right)$ we have

$$
\begin{aligned}
\mathrm{d} s_{\mathbb{P}_{2}}^{2} & =\frac{\left|\mathrm{d} x_{1}\right|^{2}+\left|\mathrm{d} x_{2}\right|^{2}+\left|x_{1} \mathrm{~d} x_{2}-x_{2} \mathrm{~d} x_{1}\right|^{2}}{\left(1+\left|x_{1}\right|^{2}+\left|x_{2}\right|^{2}\right)^{2}} \\
& \leqslant \frac{\left|\mathrm{d} x_{1}\right|^{2}}{\left(1+|x|_{1}^{2}\right)^{2}}+\frac{\left|\mathrm{d} x_{2}\right|^{2}}{\left(1+\left|x_{2}\right|^{2}\right)^{2}}+\frac{\left|\mathrm{d}\left(\frac{x_{2}}{x_{1}}\right)\right|^{2}}{\left(1+\left|\frac{x_{2}}{x_{1}}\right|^{2}\right)^{2}} .
\end{aligned}
$$

Obviously, it is sufficient to show an upper bound in terms of the degree only for a generic class of embedded curves: Let $\mathcal{C}_{d}$ be the set of all smooth plane curves $C$ of degree $d$ such that

(i) $P_{j} \notin C$ for $j=0,1,2$;

(ii) $\pi_{2} \mid C: C \rightarrow L_{2}$ is a simple branched covering.

We estimate the length of a particular class of real algebraic curves. Let $C \in \mathcal{C}_{d}$, and let $L_{\mathbb{R}} \subset L_{2}$ be a closed geodesic. We denote its preimage under $\pi_{2} \mid C: C \rightarrow$ $L_{2}$ by $C_{\mathbb{R}}$.

LEMMA 2. The curves $\pi_{0}\left(C_{\mathbb{R}}\right) \subset L_{0}$ and $\pi_{1}\left(C_{\mathbb{R}}\right) \subset L_{1}$ are real algebraic of degree at most $d^{2}$.

Proof. Let $C$ be the zero set $V(F)$ with $F=F\left(x_{0}, x_{1}, x_{2}\right)$ homogeneous and irreducible of degree $d$. We first show the claim for the $L_{\mathbb{R}}=\overline{\{(t, 1) ; t \in \mathbb{R}\}} \subset L_{2}$ and $\pi_{0}\left(C_{\mathbb{R}}\right)$ say. Since $C$ is irreducible of degree greater than one, it intersects any fiber of the map $\pi_{0}$ in a discrete set of points. Therefore we can restrict ourselves to an affine set $U=\mathbb{P}_{2} \backslash L_{1}=\pi_{0}^{-1}\left(L_{0} \backslash\left\{P_{2}\right\}\right)$. Then $\pi_{0}\left(C_{\mathbb{R}}\right)$ is the closure of $\pi_{0}\left(U \cap C_{\mathbb{R}}\right)$. Now $\left(0: 1: x_{2}\right) \in \pi_{0}\left(U \cap C_{\mathbb{R}}\right)$, if and only if there exists $t \in \mathbb{R}$ such that $F\left(t, 1, x_{2}\right)=0$. Classical elimination theory yields the following. Denote by $R\left(x_{2}, \overline{x_{2}}\right)$ the resultant of $\operatorname{Re}\left(F\left(t, 1, x_{2}\right)\right)$ and $\operatorname{Im}\left(F\left(t, 1, x_{2}\right)\right)$ with respect to $t$. We use the fact that for any two polynomials $g(y, z), h(y, z)$ of degree $m$ and $n$ resp. the resultant $R_{g, h}(z)$ (where $y$ is eliminated) is a polynomial of degree at most $m \cdot n$. Hence $\pi_{0}\left(C_{\mathbb{R}}\right)$ is real algebraic of degree at most $d^{2}$. 
Let $L_{\mathbb{R}} \subset L_{2}$ be an arbitrary geodesic. Then $L_{\mathbb{R}}$ is the closure of the set of all $(a+b \cdot t: c+d \cdot t: 0) \in L_{2} ; t \in \mathbb{R}$, where the $a, b, c, d$ determine an element of $\mathrm{SU}(2)$. As above the resultant of $\operatorname{Re}\left(F\left(a+b \cdot t, c+d \cdot t, x_{2}\right)\right)$ and $\operatorname{Im}(F(a+b \cdot$ $\left.\left.t, c+d \cdot t, x_{2}\right)\right)$ is a polynomial of degree at most $d^{2}$ in $x_{2}$ and $\overline{x_{2}}$.

LEMMA 3. Let $C_{\mathbb{R}} \subset \mathbb{P}_{1}(\mathbb{C})$ be a real algebraic curve of degree $\delta$. Then the length of $C_{\mathbb{R}}$ with respect to the Fubini-Study metric of $\mathbb{P}_{1}(\mathbb{C})$ is at most $2 \pi \delta$.

Proof. We assume that $C_{\mathbb{R}} \subset \mathbb{C} \subset \mathbb{P}_{1}$ is connected and choose a piecewise smooth parametrization $\gamma:[0,1] \rightarrow C_{\mathbb{R}} ; \gamma(t)=u(t)+i v(t)$. Then

$$
L(\gamma)=\int_{0}^{1} \frac{\left(\left|u^{\prime}\right|^{2}+\left|v^{\prime}\right|^{2}\right)^{1 / 2}}{1+u^{2}+v^{2}} \mathrm{~d} t \leqslant \int_{0}^{1} \frac{\left|u^{\prime}\right|}{1+u^{2}} \mathrm{~d} t+\int_{0}^{1} \frac{\left|v^{\prime}\right|}{1+v^{2}} \mathrm{~d} t .
$$

Since the projections $z \mapsto u$ and $z \mapsto v$, restricted to $C_{\mathbb{R}}$ have at most a number of $\delta$ sheets, the above integral is at most $2 \delta \cdot \int_{-\infty}^{+\infty}\left(\mathrm{d} u / 1+u^{2}\right)=2 \delta \pi$.

\section{Proof of the Theorem}

In the sequel, we describe a generic type of branching. Let $C \subset \mathbb{P}_{2} \backslash\left\{P_{0}, P_{1}, P_{2}\right\}$ and denote by $\pi: C \rightarrow \mathbb{P}_{1}$ the restriction of $\pi_{2}: \mathbb{P}_{2} \backslash P_{2} \rightarrow L_{2}$ to $C$. Again $\pi$ must have only simple generic branch points $P_{j}$ and we impose that

(iii) the images $Q_{j}=\pi\left(P_{j}\right)$ of the branch points $P_{j}$ are distinct, where $j=$ $1, \ldots, b$, with $b=d^{2}-d$, and no three of these are contained in a closed geodesic.

Next we choose a point $R$ in $\mathbb{P}_{1} \backslash \bigcup_{j<k} L_{j k}$, where $L_{j k}$ is the closed geodesic through $Q_{j}$ and $Q_{k}$. Let $S_{j}$ be the segment of the real projective line from $R$ to $Q_{j}, j=1, \ldots, d$, and $S=\cup S_{j}$. The complement $\mathbb{P}_{1} \backslash S$ is simply connected and $\pi^{-1}\left(\mathbb{P}_{1} \backslash S\right)$ decomposes into $b$ isomorphic copies $E_{v} ; v=1, \ldots, d$, where we set $E_{v}=\mathbb{P}_{1}^{(v)} \backslash \cup S_{j}^{(v)}$, with copies $\mathbb{P}_{1}^{(v)}$ and $S_{j}^{(v)}$ resp. of $\mathbb{P}_{1}$ and $S_{j}$ resp. Let $R^{(v)} \in \mathbb{P}_{1}^{(v)}$ correspond to $R$.

Any branch point $B_{j}$ is contained in the closure $\overline{E_{v}}$ of $E_{v}$ in $C$ for exactly two values of $v$. For all $j$ with $P_{j} \notin \overline{E_{v}}$ we fill $S_{j}^{(v)} \backslash R^{(v)}$ into $E_{v}$ and obtain $\tilde{E}_{v}$, which is a copy of $\mathbb{P}_{1}$ with a certain number of segments emanating from one point removed. We count boundary points of $\tilde{E}_{v}$ twice, except for the endpoints of line segments. The domain $\tilde{E}_{v}$ with boundary added is called $\hat{E}_{\nu}$. Now $C$ is obtained from $\cup \hat{E}_{v}$ by means of the usual gluing process. There is a natural projection $\rho_{\nu}: \hat{E}_{v} \rightarrow \mathbb{P}_{1}$. We chose arbitrary sheets $\hat{E}_{1}$ and $\hat{E}_{2}$ say and points $R_{j} \in \hat{E}_{j} ; j=1,2$ with $\rho_{j}\left(R_{j}\right)=R ; j=1,2$. We want to connect the images of $R_{1}$ and $R_{2}$ in $C$ by the images of line segments in the boundaries of $\hat{E}$, where sheets are switched at branch points. We give the construction. Let $S_{j, 1}^{(v)}, S_{j, 2}^{(\nu)} \subset \partial \hat{E}_{v}$ correspond to $S_{j}^{(v)} \subset \mathbb{P}_{1}^{(v)}$. We follow one of these segments from $R_{1}$ in $\hat{E}_{1}$ to the adjacent branch point. Either we switch sheets at the branch point, or go back on the opposite edge of the same $\hat{E}_{v}$. We follow the next edge on the present sheet to the next 
branch point and switch again sheets, or not, keeping the orientation, i.e., in a way such that the set $\hat{E}_{v}$ is always ot the same side of the edge. After circulating a certain number of times we arrive at $R_{2}$. Now we need to visit any branch point at most once: otherwise we get a closed loop which we can eliminate from our path. Hence the total number of segments does not exceed twice the number of branch points $2 b$.

In order to conclude the proof, is is sufficient to show the claim for generic $C \subset \mathbb{P}_{2}$ with $\pi: C \rightarrow \mathbb{P}_{1}$ as above. Let two points of $C$ be given. By a continuity argument one of these can play the role of $R_{2}$, whereas the other point is contained in the image of some other sheet $\hat{E}_{v}$ say $\hat{E}_{2}$ and can be connected with some $R_{2}$ located over $R_{1}$ on the corresponding boundary component. This amounts to a total of at most $2 b+1$ segments. According to Lemma 2 and 3 the length of each segment is at most $\left(4 d^{2}+1\right) \pi$, which shows that the diameter is bounded from above by $(2 b+1)\left(4 d^{2}+1\right) \cdot \pi=\left(2 d^{2}-2 d+1\right)\left(4 d^{2}+1\right) \pi$.

\section{References}

1. A'Campo, N.: On the shape of projective plane curves, Math. Res. Lett. 2 (1995), 537-539.

2. Bogomolov, F. A.: On the diameter of plane algebraic curves, Math. Res. Lett. 1 (1994), 95-98.

3. Briskin, M. and Yomdin, Y.: Semialgebraic geometry of polynomial control problems, In: Computatonal Algebraic Geometry, Progr. Math. 109, Birkhäuser, Basel, 1993, pp. 21-28.

4. Gromov, M.: Curvature and Betti numbers, Comment. Math. Helv. 56 (1981), 179-195.

5. Ness, L.: Curvature on algebraic plane curves I, Compositio Math. 35 (1977), 57-63.

6. Yomdin, Y.: The geometry of critical and near-critical values of differential mappings, Math. Ann. 264 (1983), 495-515. 